\title{
GLPSobControle: um jogo sério para o treinamento operacional de controle de vazamento de gás de cozinha
}

\author{
Rafaela Vilela da Rocha ${ }^{1}$, Marcio Roberto de Campos ${ }^{2}$ \\ ${ }^{1}$ Departamento de Computação - Universidade Federal de São Carlos (UFSCar) \\ CEP 13565-905 - São Carlos -SP - Brasil \\ ${ }^{2}$ Departamento de Engenharia de Produção - Universidade Federal de São Carlos \\ (UFSCar) - CEP 13565-905 - São Carlos -SP - Brasil \\ \{rafaela.vilela, marcamposbr\}@gmail.com
}

\begin{abstract}
Resumo. GLPSobControle é um jogo sério, composto de 7 fases baseadas na taxonomia de Bloom, para treinamento do protocolo operacional de controle de vazamento de gás de cozinha. Ele foi criado utilizando uma metodologia iterativa e integradora de desenvolvimento de jogos sérios e avaliado com o apoio do Corpo de Bombeiros do Estado de São Paulo. Os resultados indicam que ele possibilita o treinamento das competências intencionada. Além disto, houve a percepção na necessidade de treinamento constante e a simulação permitiu avaliar uma oportunidade de melhoria no protocolo operacional utilizado.
\end{abstract}

\section{Cenário de Uso}

Os treinamentos práticos tradicionais, em geral, têm custos elevados, ocorrem esporadicamente e podem ter riscos reais de acidentes (danos ao meio ambiente, á vida e ao patrimônio). Além de que, muitas vezes não há uma avaliação sistemática e padronizada do desempenho dos participantes (Green III, 2000; Salas e Rosen, 2007). Por outro lado, o treinamento com jogo sério é oportuno para superar estas dificuldades, pois reproduz a realidade em um ambiente virtual seguro, pode ser jogado várias vezes, por diferentes pessoas, além de possibilitar um aprendizado experiencial, sistemático e estruturado, somado ao fato de apoiar a avaliação e o feedback contínuo (Mattar, 2010; Salas et al., 2009).

Neste contexto, este artigo descreve um jogo sério criado, cujo público-alvo são os bombeiros do Corpo de Bombeiros da Polícia Militar do Estado de São Paulo (CB/PMESP), aproximadamente $10 \mathrm{mil}$, e os profissionais de outras empresas e instituições afins. O treinamento selecionado foi o uso do protocolo de controle de vazamento de gás de cozinha (Gás Liquefeito de Petróleo - GLP) para controlar, ventilar, salvar vítimas e deixar o local de vazamento em segurança (São Paulo, 1997a e 1997b). Ele foi selecionado pois o índice de ocorrência deste tipo de emergência é alto no Estado de São Paulo, com média de atendimento de 12 ocorrências por dia no estado.

\section{Desenvolvimento}

O GLPSobControle foi desenvolvido utilizando a metodologia iterativa e integradora, nomeada DevJSTA, para o desenvolvimento de Jogos Sérios para Treinamento e Avaliação do desempenho humano (JSTA), descrita em Rocha (2014). Ela provê desde orientações e artefatos até elementos de jogos e modelos de simulação, para integrar e 


\section{CBIE-LACLO 2015}

Anais dos Workshops do IV Congresso Brasileiro de Informática na Educação (CBIE 2015)

inserir conceitos de aprendizagem efetiva e experiencial aos jogos sérios, além de avaliação do desempenho humano e fornecimento de feedback durante e pós-ação. Ela é composta de oito processos: (1) planejamento, (2) análise, (3) projeto, (4) implementação, (5) integração e testes, (6) execução do treinamento e (7) avaliação, que são sequenciais, porém iterativos; e o processo de (8) verificação e validação que é realizado ao final de cada processo.

O JSTA GLPSobControle foi planejado para treinar as seguintes competências: (1) Conhecimentos: conhecer o protocolo de controle de vazamento de GLP; (2) Habilidades técnicas operacionais: usar corretamente o protocolo de controle de vazamento de GLP para controlar, ventilar, salvar vítimas e deixar o local de vazamento de GLP em segurança; (3) Atitudes: garantir o acesso seguro, controlar e extinguir o vazamento de GLP, ventilar o ambiente e deixá-lo em segurança.

O contexto e o procedimento de vazamento de gás de cozinha foram analisados e projetados nas fases do jogo sério, por meio dos modelos integradores descritos na metodologia DevJSTA (Rocha e Araujo, 2013). O modelo de integração programa de treinamento- avaliação possibilitou incluir diferentes atividades e tipos de avaliação (Miller, Imrie e Cox, 1998; Piskurich, Beckschi e Hall, 2000; Salas et al., 2009; Kirkpatrick e Kirkpatrick, 2006) e feedback (Salas et al., 2009; Salas e Rosen, 2007) ao longo do processo de treinamento, com o uso de diferentes recursos (simulações 3D, jogos simples 2D, vídeos e um formulário de avaliação). Estes requisitos são contemplados utilizando teorias e trabalhos correlatos que possibilitam explorá-los na criação das fases, conforme os critérios apresentados no Quadro 1.

Segundo o modelo integrador programa de avaliação- treinamento (Rocha, 2014), as fases 1 e 2 devem explorar o nível 1 da taxonomia de Bloom, descrevendo objetivos que possibilitem lembrar o protocolo treinado. A fase 1 deve ser diagnóstica e possibilitar o aprendiz fazer as ações a partir de conhecimentos prévios e ver/sentir os resultados (experiência concreta); ao passo que a fase 2 deve incluir atividades que permitam a comparação e generalização de fatos e elementos da realidade (conceitualização abstrata) e fornecer feedback prescritivo além do descritivo. As atividades nas fases 3 e 4 devem possibilitar o aprendiz planejar e fazer ações a partir dos conhecimentos refletidos (experiência ativa). Nelas, o aprendiz deve poder compreender e aplicar estes conhecimentos e obter feedback prescritivo (como deveria ter realizado) além do descritivo (acertos e erros). As fases 5 e 6 devem possibilitar uma avaliação somativa, e o aprendiz deve analisar e sintetizar os conhecimentos praticados a partir da observação e reflexão. A última fase compreende a avaliação por parte do aprendiz. Ele deve avaliar o seu treinamento e o programa de treinamento em si.

Após as fases de planejamento, análise e projeto, o jogo sério foi implementado, integrado e testado com o uso do motor de jogos Unity $3 D(\mathbb{C}$, em conjunto a linguagem C\# para programação da simulação. Modelos 3D foram reusados a partir do repositório do 3 DWarehouse $\mathbb{C}$. Foram criadas animações simples para as portas e o quadro de energia, e usados sistemas de partículas do próprio Unity $3 D$ para representar a explosão e o vazamento de gás. Áudios e texturas foram reusadas de repositórios gratuitos encontrados na Internet. O banco de dados foi desenvolvido em linguagem MySQL, com acesso implementado em PHP. O questionário de avaliação (fase 7) foi criado com o Google Docs@. 
CBIE-LACLO 2015

Anais dos Workshops do IV Congresso Brasileiro de Informática na Educação (CBIE 2015)

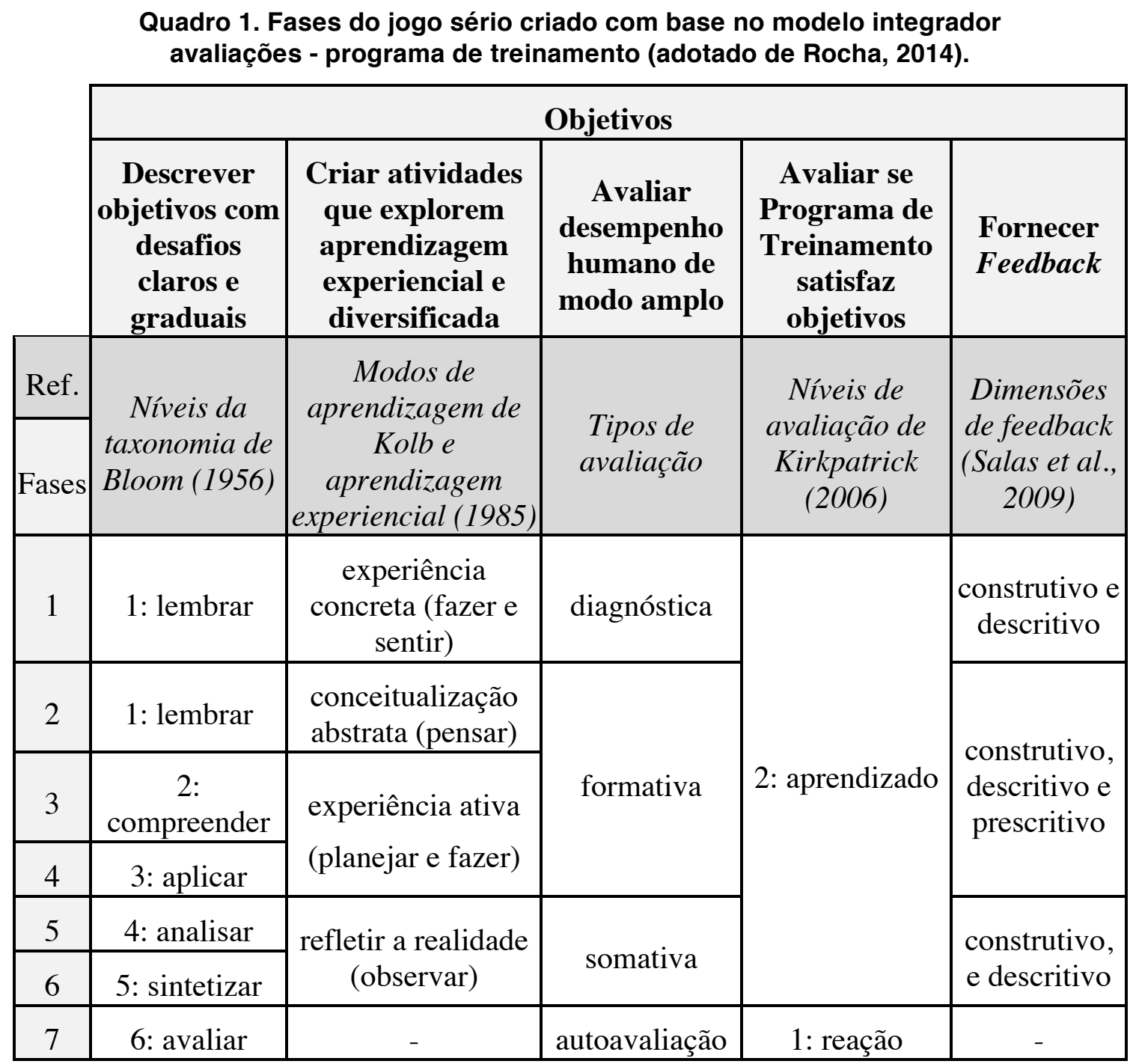

O JSTA GLPSobControle foi validado e avaliado pelos bombeiros, no que tange aos aspectos de fidelidade do modelo de simulação e conteúdo, aspectos educacionais de desempenho humano e treinamento das competências intencionadas, bem como a percepção disto pelos aprendizes.

Em um primeiro momento, foram feitas avaliações e validações por dois oficiais bombeiros: do modelo de simulação de controle de vazamento de GLP; do jogo completo GLPSobControle; e do questionário utilizado para avaliação da reação do aprendiz e autoavaliação. As validações foram exitosas, inclusive possibilitaram verificar uma oportunidade de melhoria no protocolo que não tinha sido observada anteriormente (somente com o documento que descreve o procedimento). Isto ocorre quando a vítima e o recipiente de gás estão próximos ou o recipiente é alcançado antes da vítima. No protocolo, a sequência de ações é salvar vítimas e depois cortar o fluxo de gás. Além disto, é descrita a prioridade de remover a vítima antes de qualquer outra ação. Entretanto, quando as situações descritas anteriormente acontecem, é mais seguro cortar o fluxo de gás antes de salvar as vítimas. Dessa forma, esta sequência foi alterada no modelo de simulação e será atualizada no protocolo do Corpo de Bombeiros.

Depois disto, os treinamentos foram realizados com uma amostra intencional de 


\section{CBIE-LACLO 2015}

Anais dos Workshops do IV Congresso Brasileiro de Informática na Educação (CBIE 2015)

oito bombeiros (que abrange os perfis que utilizarão o jogo validado). Quanto a validade do conteúdo pelos aprendizes, ela foi realizada por meio de um questionário e de entrevistas abertas informais. Esta validação tem dois objetivos: (1) validar a representação física e comportamental da simulação criada (artefato final criado); e (2) verificar se os aprendizes conseguem relacionar o treinamento à realidade, por meio da autoavaliação. Após a realização do treinamento e coleta de dados (por meio do jogo sério e do questionário), os resultados foram organizados e analisados (conforme descrito em Rocha, 2014).

Segundo o método de transferência inversa descrito por Korteling, Oprins e Kallen (2009), o programa de treinamento é válido e tem efetividade educacional externa se profissionais experientes na prática conseguem utilizar o jogo sério com sucesso. Metade dos profissionais que realizaram o treinamento com sucesso são praças (cabos, soldados e sargentos) que tem mais de 10 anos de experiência e atenderam mais de cinco vazamentos de GLP. Estes profissionais concordam que conseguem relacionar o que treinaram com a realidade e que o treinamento contribuiu para relembrar e praticar o protocolo. Eles ressaltaram também a necessidade de treinamento constante pois são muitos os Procedimentos Operacionais Padrão e com o tempo os detalhes dos procedimentos acabam esquecidos.

Dessa forma, o pressuposto é que se os profissionais experientes conseguiram utilizar e relembrar o protocolo, então a transferência ocorrerá com os aprendizes que completaram com sucesso este treinamento e com outros que o utilizarem com a supervisão e auxílio do instrutor. Assim, o JSTA GLPSobControle tem indícios de possuir validade educacional externa. Entretanto, é necessário a sua aplicação em novos casos e o acompanhamento dos desempenhos dos aprendizes durante um tempo após o treinamento para a comprovação dos efeitos imediatos e a longo prazo para a corporação e assim a confirmação desta validade.

\section{Apresentação do Jogo Sério}

O jogo sério GLPSobControle é composto de sete fases e pode ser jogado gratuitamente no site http://glpsobcontrole.url.ph, onde também está disponível o vídeo demonstrativo. O objetivo da primeira fase é diagnosticar o conhecimento prévio do jogador, o qual deve clicar e arrastar cartas, conforme ilustradas na Figura 1, para responder três questões: (1) Qual é a sequência básica para controlar um vazamento de GLP? (Figura 1); (2) Se o quadro de força estiver na cozinha da residência, próximo do vazamento de GLP, o que deve ser feito?; e (3) Em um vazamento de GLP, se tiver vítimas e incêndio, o que você deve fazer primeiro?

O objetivo da segunda fase é que o jogador relembre ou aprenda o protocolo básico. Ele deve seguir as orientações das ações, que são ilustradas sequencialmente nas cartas 2D (posicionadas na parte superior da tela), e descritas na parte inferior (conforme ordem de execução). Estas ações são realizadas por meio de cliques nos objetos em um ambiente virtual 3D, conforme Figura 2. Também há orientações de cuidados a tomar durante a realização do procedimento operacional (cartas à esquerda).

O objetivo da terceira fase é verificar se o jogador compreendeu o uso do protocolo básico. Ele deve realizar as ações do protocolo no mesmo cenário da fase 2 , porém, sem orientações. 


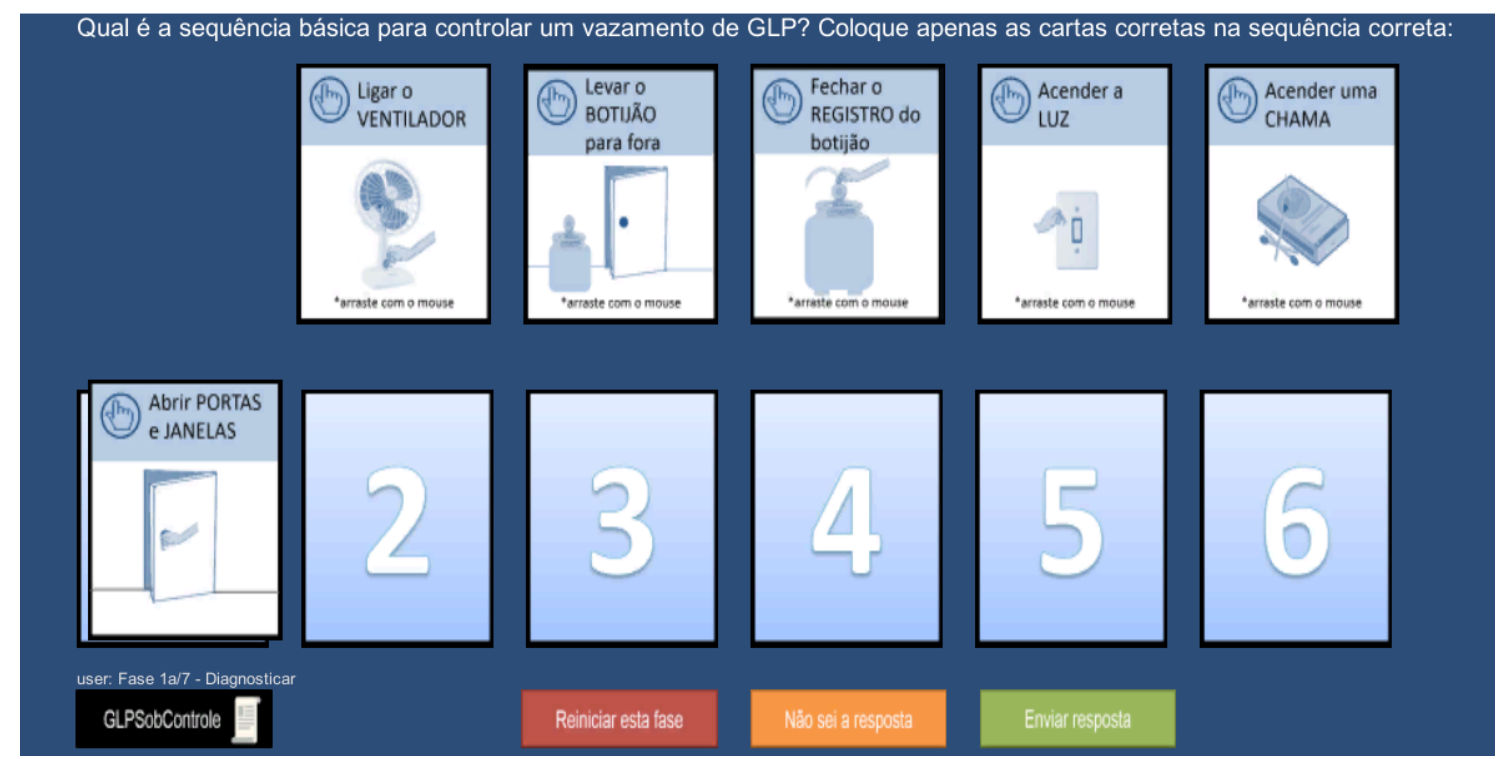

Figura 1. Primeira fase: diagnosticar.

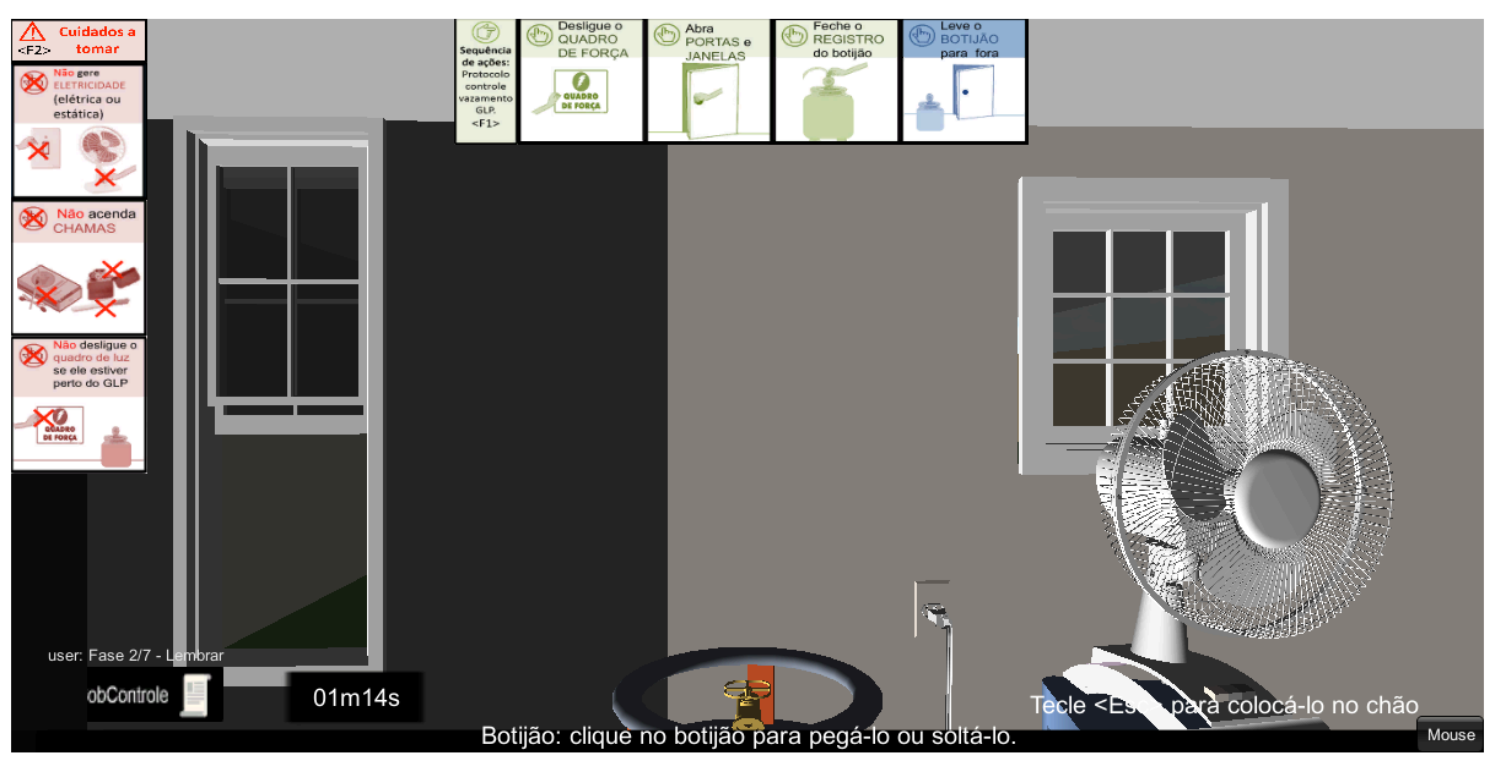

Figura 2. Segunda fase: lembrar.

A quarta fase possui um novo cenário, conforme pode ser visto na Figura 3, que é jogado do mesmo modo que a segunda e terceira fases. O objetivo é que o jogador aplique e treine o que foi aprendido sobre o uso do protocolo, sem orientações e em um outro cenário que possui um novo desafio: salvar uma vítima que está próxima ao botijão de gás e ao quadro de força.

Na quinta fase, o jogador assiste a um vídeo em que outra pessoa realiza o protocolo treinado. O objetivo é que ele analise as ações gravadas e identifique se elas foram realizadas incorretamente, conforme Figura 4.

O objetivo da sexta fase é que o jogador sintetize o seu conhecimento combinando sentenças sobre o protocolo treinado. Para isto, ele arrasta as cartas para as casas inferiores, de modo a combinar a descrição de cada casa com a carta sobreposta, conforme apresentado na Figura 5. 
CBIE-LACLO 2015

Anais dos Workshops do IV Congresso Brasileiro de Informática na Educação (CBIE 2015)

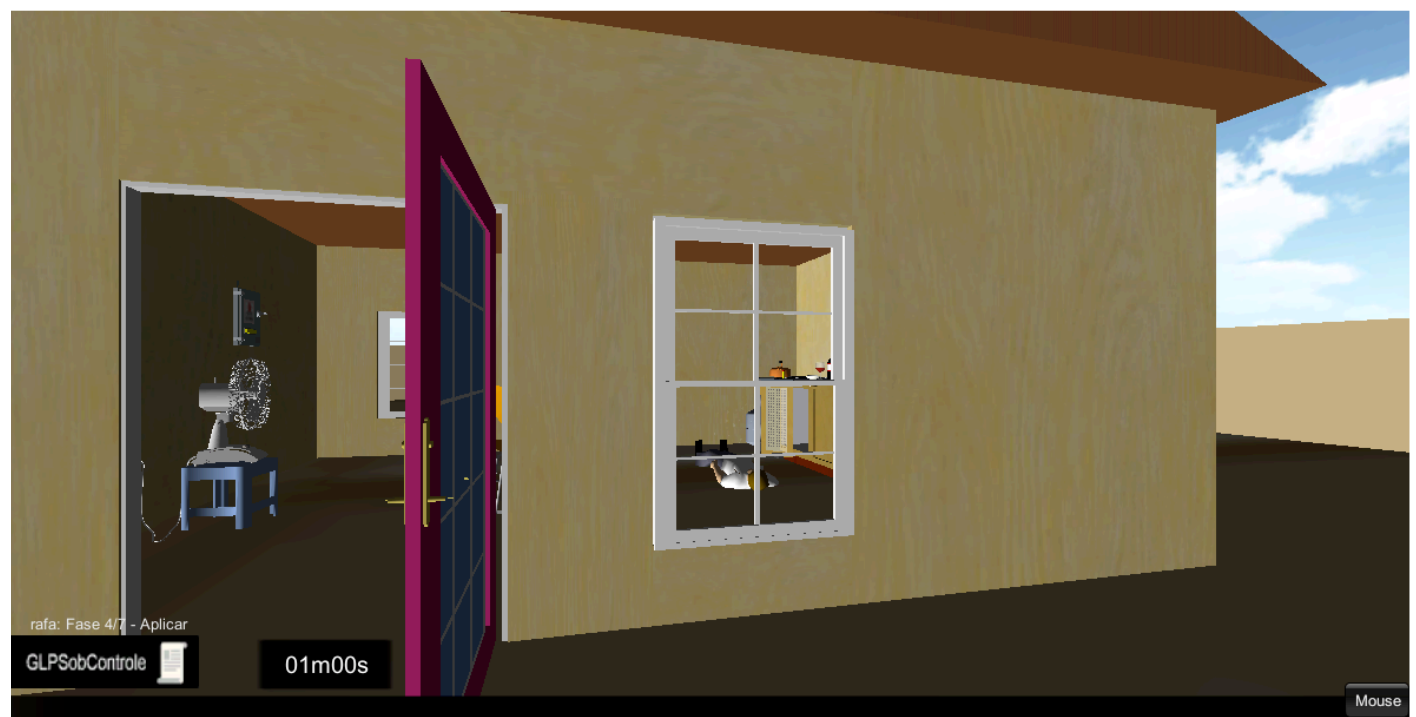

Figura 3. Quarta fase: aplicar.

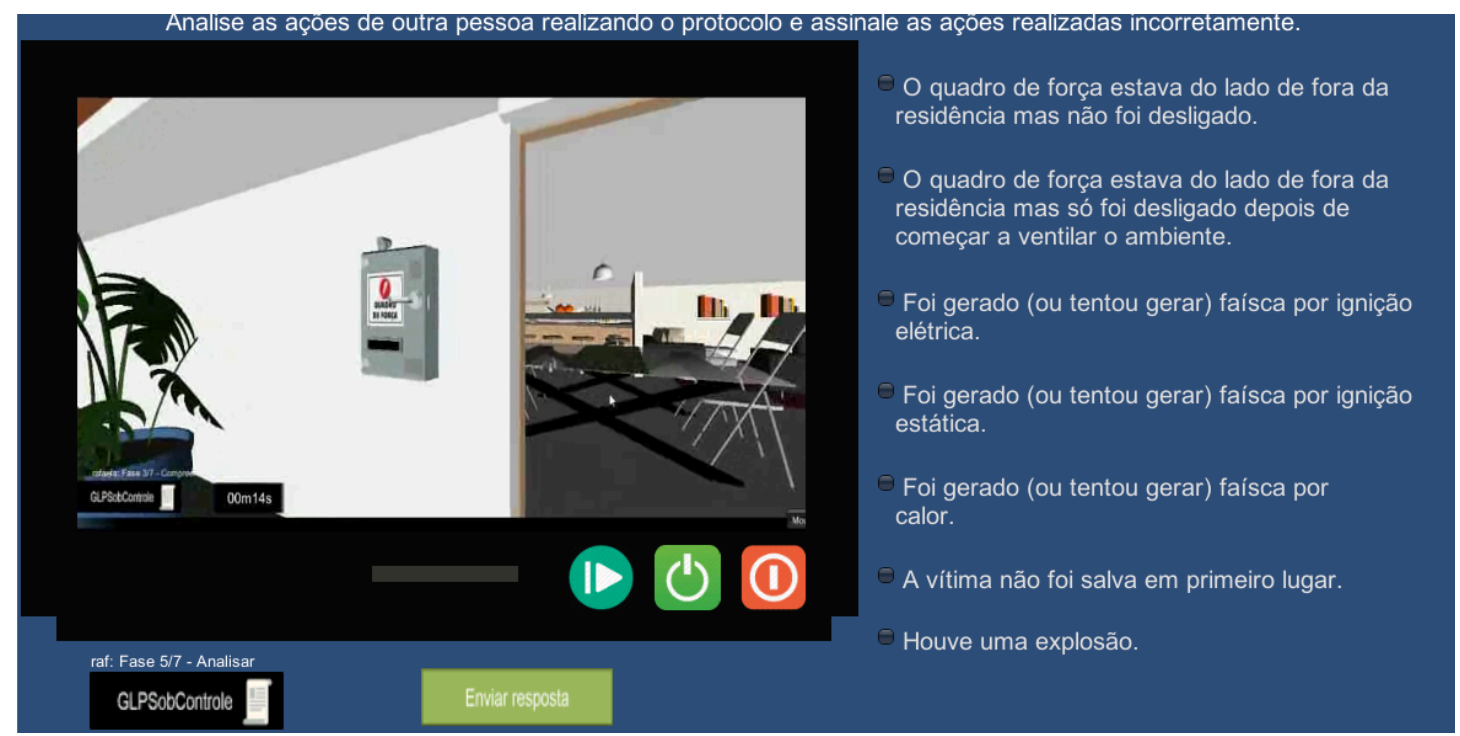

Figura 4. Quinta fase: analisar.

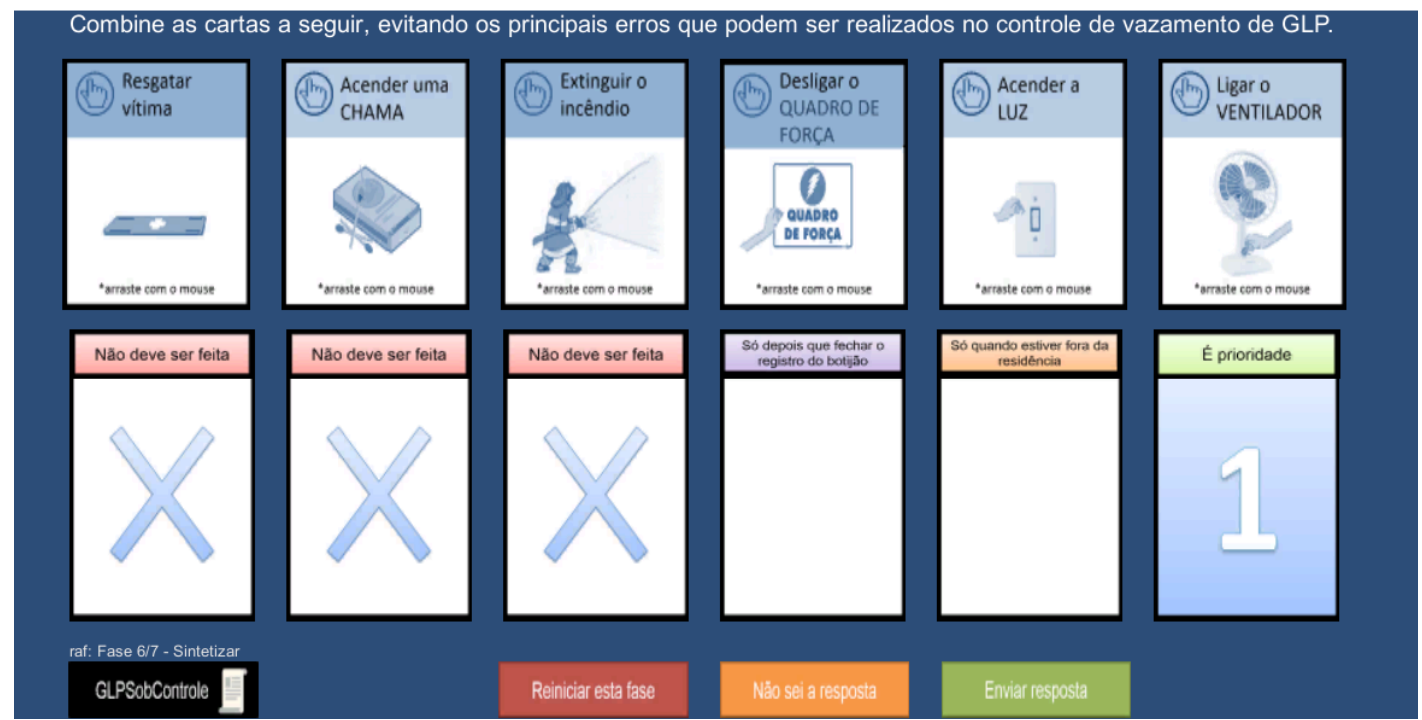

Figura 5. Sexta fase: sintetizar. 


\section{CBIE-LACLO 2015}

Anais dos Workshops do IV Congresso Brasileiro de Informática na Educação (CBIE 2015)

O objetivo da sétima e última fase é que o jogador se auto-avalie (avalie o seu desempenho no treinamento) e avalie também o jogo sério, por meio de um questionário (dividido em 4 tópicos: jogo; simulação; aprendizagem com o jogo; e aprendizagem durante as fases) que contém 47 afirmações avaliativas fechadas (agrupadas em subtópicos), 16 espaços para comentários, uma questão fechada e quatro abertas (Rocha, 2014). Essas 47 afirmações devem ser avaliadas e pontuadas de 1 a 5 na escala Likert, com um ponto central de neutralidade (Pereira, 2004) (julgamento 1: discordo totalmente, 2: discordo, 3: neutro, 4: concordo, 5: concordo totalmente). A questão fechada e obrigatória é subdividida em sete itens no qual o aprendiz deve pontuar cada uma das fases de 1 a 10 de acordo com sua satisfação. Cada subtópico (que contém uma ou mais afirmações, ou a questão fechada) contém um espaço para comentário. O questionário é finalizado com 4 questões abertas. O questionário foi validado semanticamente por especialistas no domínio (Rocha, 2014).

\section{Considerações Finais}

Os resultados indicam que o jogo sério GLPSobControle possibilita o treinamento das competências intencionada. Além disto, houve a percepção na necessidade de treinamento constante e a simulação permitiu avaliar uma oportunidade de melhoria no protocolo operacional utilizado. Para confirmar sua validade educacional, ele será utilizado em novos estudos de caso, e será feito o acompanhamento dos desempenhos dos aprendizes durante um tempo após o treinamento, para a comprovação dos efeitos imediatos e em longo prazo para a corporação.

Como trabalhos futuros, deverão ser melhoradas as interfaces gráficas; adicionados mais atividades em cada fase, com por exemplo, outros tipos de botijões, gás encanado, incêndio em GLP; além de novos cenários (tais como, favelas, condomínios, comércios, indústrias). Além disto, projetos futuros poderão incluir o reconhecimento do nível de usuário e seu conhecimento, para personalizar a fase e a quantidade de informações, explicações e desafios que são oferecidos.

\section{Agradecimentos}

Este projeto foi financiado pelo CNPq (Processo 480291/2012-8), FAPESP e CAPES (Projeto INCT-SEC, processos 2010/07179-8, 08/57870-9, 237216/2012-4, 143567/2011-0, 573963/2008-8). Os autores gostariam de agradecer ao Corpo de Bombeiros por seu apoio.

\section{Referências}

Bloom, B.S. (1956). Taxonomy of Educational Objectives: the classification of educational goals - Handbook I: Cognitive Domain. New York: McKay.

Green III, W. G. (2000). Exercise Alternatives for Training Emergency Management Command Center Staffs. Command Center Staffs: Universal Publishers.

Kirkpatrick, D.L.; kirkpatrick, J.D. (2006). Evaluating Training Programs: The Four Levels. 3rd Edition. San Francisco, CA, USA: Berrett-Koehle. 568 p.

Kolb, A.Y.; Kolb, D.A. (2005). Learning Styles and Learning Spaces: Enhancing Experiential Learning in Higher Education. Academy of Management Learning and Education, v. 4, n. 2.p. 193-212. 


\section{CBIE-LACLO 2015}

Anais dos Workshops do IV Congresso Brasileiro de Informática na Educação (CBIE 2015)

Kolb, D.A. (1984). Experiential Learning: Experience as The Source of Learning and Development. Englewood Cliffs: Prentice-Hall Inc. p. 20-38.

Korteling, J.E., Oprins, E.A.P.B.; Kallen, V.L. (2012). Measurement of Effectiveness for Training Simulations. In: RTO System Analysis and Studies Panel (SAS), RTOMP-SAS-095. Amsterdam, The Netherlands. p. 1-14.

Mattar, J. (2010). Games em Educação: como os nativos digitais aprendem. São Paulo: Pearson Prentice Hall. 181 p.

Miller, A.; Imrie, B.W.; Cox, K. (1998). Functions of Assessment. In: Student Assessment in Higher Education: a handbook for assessing performance. London: Kogan Page. p. 23-40.

Pereira, J.C.R. (2004). Análise de Dados Qualitativos: estratégias metodológicas para as Ciências da Saúde, Humanas e Sociais. 3a. ed. São Paulo: EDUSP. 160 p.

Piskurich, G.; Beckschi P.; Hall, B. (2000). The ASTD Handbook of Training Design and Delivery: a comprehensive guide to creating and delivering training programs: instructor-led, computer-based, or self-directed. 2nd ed., New York: McGraw-Hill.

Rocha, R.V. (2014). Metodologia iterativa e modelos integradores para desenvolvimento de jogos sérios de treinamento e avaliação de desempenho humano. Tese (Doutorado em Ciência da Computação) - Departamento de Computação, Universidade de São Carlos, São Carlos - SP, 2014.

Rocha, R.V.; Araujo, R.B. (2013). Metodologia de Design de Jogos Sérios para Treinamento: Ciclo de vida de criação, desenvolvimento e produção. In: Simpósio Brasileiro de Jogos e Entretenimento Digital, São Paulo. p. 63-72.

Salas, E.; et al. (2009). Performance Measurement in Simulation-Based Training: A Review and Best Practices, In: Simulation Gaming, v. 40, n. 3. p. 328-376.

Salas, E.; Rosen, M.A. (2007). Best Practices for Performance Measurement in Military Simulation-based Training: observations from the field. Report - Department of Psychology, Institute for Simulation and Training, University of Central Florida.

São Paulo (Estado). Polícia Militar. Corpo de Bombeiros. (1997a). Incêndio em GLP. Procedimento Operacional Padrão POP-INC-002. São Paulo. 1 p.

São Paulo (Estado). Polícia Militar. Corpo de Bombeiros. (1997b). Incêndio em GLP. Caderno de Treinamento do POP-INC-002. São Paulo. 18 p. 